\title{
Soluções para o transporte urbano de cargas na etapa de última milha
}

\author{
Solutions for last mile deliveries
}

Camilla de Oliveira Souza[a] (1), Márcio de Almeida D'Agosto[a] (ㄱ,

Renata Albergaria de Mello Bandeira[b] @

[a] Universidade Federal do Rio de Janeiro (UFRJ), Programa de Engenharia de Transportes, Ilha do Fundão, RJ, Brasil

[b] Instituto Militar de Engenharia, Programa de Pós-graduação em Engenharia de Transportes, Praia Vermelha, RJ, Brasil

Como citar: Souza, C. O., D’Agosto, M. A., Bandeira, R. A. M., \& Almeida, I. R. P. L. (2020). Soluções para o transporte urbano de cargas na etapa de última milha. urbe. Revista Brasileira de Gestão Urbana, 12, e20190138. https://doi.org/10.1590/2175-3369.012.e20190138

\section{Resumo}

Novas formas de operação na distribuição de carga urbana podem levar a melhorias na eficiência das entregas de última milha, além de reduzir seu custo e seus impactos socioambientais. Entretanto, a viabilidade financeira dessas novas formas de operação pode ainda ser questionável por causa dos altos investimentos necessários e do elevado custo de mão de obra, especialmente em países em desenvolvimento. Portanto, este trabalho tem o objetivo de avaliar como essas novas modalidades podem ser adotadas no contexto de países em desenvolvimento, mais especificamente no Brasil, identificando os principais desafios, os benefícios e o impacto dessas práticas para as cidades, as quais, muitas vezes, já sofrem com outros problemas de infraestrutura de transportes.

Palavras-chave: Transporte urbano de carga. Logística urbana. Entregas de última milha.

\section{Abstract}

New forms of operation in urban freight distribution can lead to improvements in the efficiency of last mile deliveries, besides contributing to reduce their costs and to reduce their socio-environmental impacts. However, the financial viability of such forms of operation may still be questionable due to the high investments required and the high labor cost involved, especially in developing countries. Therefore, this paper aims to assess how these new forms of operation can be implemented in the context of developing countries, identifying the main challenges, benefits and impact of these practices for the cities, which often suffer from other problems of transport infrastructure.

Keywords: Urban freight transportation. Urban logistics. Last mile deliveries.

COS é engenheiro civil, e-mail: c.souza2611@poli.ufrj.br

MADA é doutor, e-mail: dagosto@pet.coppe.ufrj.br

RAMB é doutora, e-mail: re.albergaria@gmail.com

IRPLA é mestre, e-mail: isabelarochapombo@poli.ufrj.br 


\section{Introdução}

A população urbana mundial vem aumentando ao longo dos anos, e, atualmente, 54\% dela vive em cidades, sendo esperado aumento para 66\% até 2050 (United Nations, 2014). Essa crescente urbanização leva à maior demanda por transporte de passageiros e de cargas nas cidades. Além disso, mudanças no padrão de consumo da sociedade moderna, geradas com o advento do comércio eletrônico e de práticas logísticas, como o just in time, também acarretam um aumento no número de viagens no transporte urbano de carga (TUC) (Comi \& Nuzzolo, 2016).

O TUC é uma importante fonte de emprego e facilitador do crescimento econômico nas cidades (Lindholm \& Behrends, 2012). Porém, gera impactos econômicos, sociais e ambientais relacionados ao congestionamento do tráfego, geração de ruído, consumo de energia não renovável, emissões de poluentes atmosféricos (PA) e de gases de efeito estufa (GEE), além de maior risco de acidentes de trânsito (McKinnon et al., 2015; Muñoz-Villamizar et al., 2017). Para mitigar essas externalidades, é necessário avançar na transformação das cidades, explorando inovações nas operações do TUC, e adaptar as atividades logísticas às exigências do desenvolvimento sustentável.

Ainda, a última milha (last mile) do TUC (a última etapa da entrega de uma mercadoria, ou seja, entrega ao consumidor final) representa cerca de $50 \%$ do custo total das entregas (Roumboutsos et al., 2014). Segundo Joerss et al. (2016), o custo logístico global na entrega de última milha chega a 70 bilhões de euros por ano. Em vista disso, os operadores de transporte são encorajados a minimizar seus custos de transporte e a manter um nível satisfatório de serviço, enquanto tentam reduzir o impacto social, ambiental e econômico de suas operações de transporte urbano de carga (Sanchez Rodrigues et al., 2015).

Joerss et al. (2016) destacam que o modelo de negócios atualmente aplicado à última milha pode ser descontinuado por causa de novas tecnologias que chegam ao mercado. Para esses autores, a distribuição de mercadorias tradicional, com veículos leves a diesel, será responsável por apenas 20\% das entregas de última milha em áreas urbanas. Winkenbach et al. (2016) também evidenciam o progresso vivenciado nos dias de hoje em entregas de última milha com o uso de veículos elétricos e empresas de distribuição de crowdsourcing, que são considerados modelos inovadores.

Portanto, existe potencial para que as novas formas de operação na distribuição de carga melhorem a eficiência das entregas de última milha, além de reduzir seu custo e seus impactos socioambientais. Isso pode levar a mudanças na infraestrutura de transporte e no comportamento dos clientes. Entretanto, a viabilidade financeira de algumas dessas novas formas de operação pode ainda ser questionável em razão dos altos investimentos necessários e do elevado custo de mão de obra. Assim, é possível que a adoção antecipada dessas novas formas de operação se concentre em países desenvolvidos (Joerss et al., 2016). Em países em desenvolvimento, as mudanças disruptivas sofrem resistência por causa da necessidade de altos investimentos em novas tecnologias para a troca de informações, veículos e equipamentos e para a qualificação de profissionais.

Nesse contexto, é essencial identificar novas formas de operação adotadas na última milha, bem como compreender melhor os requisitos necessários para sua adoção. Assim, este artigo busca avaliar como elas podem ser introduzidas no contexto de países em desenvolvimento, mais especificamente no Brasil, identificando os principais desafios, os benefícios e o impacto dessas práticas para as cidades, as quais, muitas vezes, já sofrem com outros problemas de infraestrutura de transportes.

0 artigo é estruturado em seis seções, além desta primeira. A seção 2 trata das novas formas de operação de última milha. Na seção 3, é descrita a metodologia adotada neste estudo, revisão bibliográfica sistemática (RBS) e pesquisa survey. A seção 4 apresenta os resultados da pesquisa, enquanto, na seção 5, é realizada a análise deles considerando o contexto de um país em desenvolvimento (Brasil). Enfim, a seção 6 apresenta a conclusão do trabalho e as recomendações para pesquisas futuras. 


\section{Novas formas de operação de última milha}

Para uma abordagem preliminar sobre o tema, esta seção se fundamenta no estudo de Joerss et al. (2016). Segundo os autores, a entrega tradicional, como é conhecida hoje, será responsável por apenas $20 \%$ da distribuição de mercadorias, continuando a ser adotada para casos especiais, como entrega de alimentos, pois as pessoas ainda querem que o transporte seja feito até suas residências. Assim, com base em uma pesquisa desenvolvida com 4.700 entrevistados na China, na Alemanha e nos Estados Unidos, Joerss et al. (2016) apresentam o atual modelo de última milha, além de propor seis modelos inovadores (Tabela 1), que serão introduzidos nos próximos dez anos em países desenvolvidos.

Joerss et al. (2016) reforçam que a velocidade com que as iniciativas apresentadas serão adotadas depende das preferências do público, regulamentação e custos trabalhistas. Os autores destacam que as iniciativas apresentadas até agora são válidas para países com custos de mão de obra acima 12 euros por hora, o que abrange praticamente todo o mundo desenvolvido. Em locais com custos de mão de obra abaixo desse limite, a entrega pelo modelo atual ainda será predominante por mais alguns anos.

Para utilizar os modelos citados nesta seção, é necessário haver alto investimento em tecnologia e qualificação de profissionais. Assim, os novos modelos de entrega serão concentrados em países desenvolvidos, onde os custos trabalhistas são altos o suficiente para justificar o investimento. Em países em desenvolvimento, os custos trabalhistas provavelmente permanecerão baixos, inviabilizando grandes mudanças tecnológicas na etapa de última milha nos próximos dez anos (Joerss et al., 2016).

Em complemento ao trabalho de Joerss et al. (2016), busca-se verificar outras formas de operação para a última milha, além de identificar os benefícios e os desafios de cada prática. Para tanto, foi realizada uma revisão bibliográfica sistemática (RBS), que será apresentada na seção 3. Além disso, também foi realizada uma pesquisa survey para compreender de que modo essas práticas podem se adaptar ao contexto de um país em desenvolvimento, como o Brasil, já que a maioria dos estudos é feita em países desenvolvidos.

Tabela 1 - Modelo de entrega de última milha

\begin{tabular}{cc}
\hline Modelo de entrega & Caracterização \\
\hline Modelo atual & Um entregador recolhe as encomendas em um ponto de consolidação, geralmente um \\
depósito, e realiza as entregas diretamente ao consumidor. Na maioria das vezes, são \\
utilizadas vans (veículos comerciais leves de peso bruto total - PBT 3,5 ) movidas a diesel. \\
Aeronaves autônomas de partida vertical que transportam até $15 \mathrm{~kg}$ de carga a seu \\
destino. Percorrem o trajeto mais retilíneo possível e com velocidade média relativamente \\
alta. É uma solução adequada para entrega de pequenos volumes, especialmente em \\
áreas rurais por causa da grande distância que deve ser percorrida para acessar o \\
consumidor. Porém, apresenta duas desvantagens: atualmente transportam, na prática, \\
cargas com até 5kg e, mesmo que a capacidade de carga alcançasse os 15 kg, ainda \\
seria inviável o transporte de mais de $5 \%$ das encomendas atuais (pois possuem peso entre \\
15 kg e 30 kg). A segunda desvantagem é que os drones apresentam grandes dimensões, \\
necessitando de áreas de pouso de pelo menos 2 m², o que pode restringir seu uso em \\
locais com alta densidade populacional. Com isso, é reforçada a ideia de adotá-los em \\
áreas rurais, com baixa densidade populacional, para encomendas que precisam ser \\
entregues no mesmo dia e com baixo peso. \\
Consiste em uma rede de motoristas, na qual praticamente qualquer pessoa habilitada \\
pode participar, cadastrando-se em uma plataforma e, assim, começando a realizar \\
entregas. A vantagem desse modelo é sua flexibilidade no fornecimento do serviço, que \\
pode ser com carros particulares ou por convênios com cooperativas de táxi. É ideal para \\
atender a uma demanda flutuante, por exemplo, uma maior necessidade desse serviço em \\
períodos próximos a datas festivas. Com isso, é possível fornecer um serviço mais barato para \\
o consumidor em razão do menor custo com trabalho regulamentado. Além disso, é um \\
modelo que apresenta baixo investimento inicial, pois os motoristas são os donos dos seus \\
próprios veículos. No entanto, pode apresentar problemas de qualidade e confiabilidade. \\
Como os motoristas geralmente se inscrevem em várias empresas de crowdsourcing, às \\
vezes pode ser difícil garantir capacidade suficiente em períodos de pico.
\end{tabular}


Tabela 1 - Continuação...

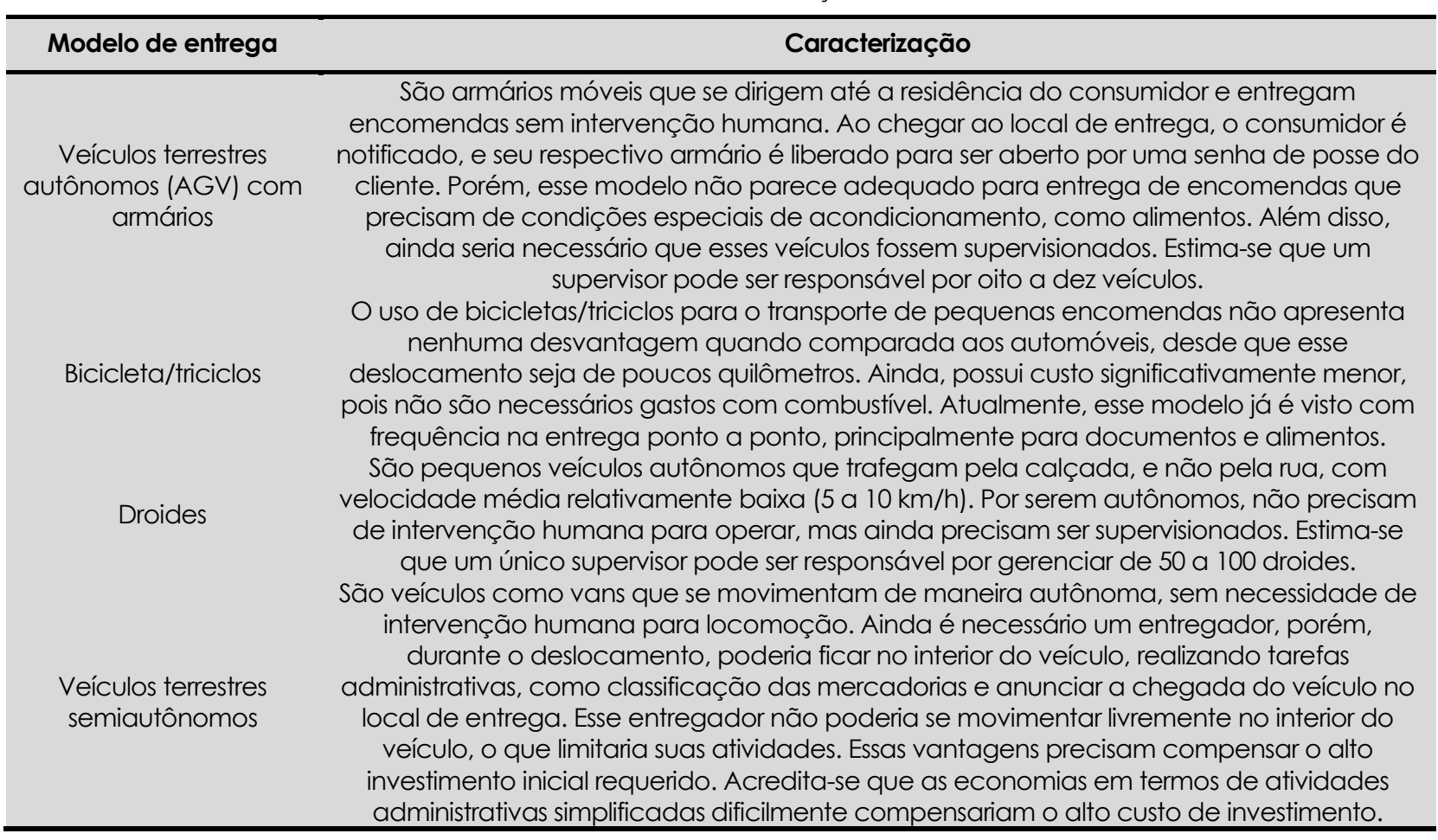

Fonte: Adaptada de Joerss et al. (2016).

\section{Metodologia}

O objetivo deste estudo consiste em avaliar como novas formas de operação de entregas de última milha podem ser adotadas no contexto de um país em desenvolvimento. Assim, em um primeiro momento, foi feita a identificação, por meio de uma revisão bibliográfica sistemática de abrangência internacional, dessas novas formas de operação. Em seguida, os resultados foram complementados, direcionando-os para a realidade dos países em desenvolvimento, em especial o Brasil, para identificar os principais desafios, benefícios e impacto dessas práticas para as cidades, as quais, muitas vezes, já sofrem com outros problemas de infraestrutura de transportes.

Para o desenvolvimento da RBS, foi adotada a metodologia proposta por Thomé et al. (2016). A pesquisa foi realizada na base de dados Web of Science, por causa da extensa literatura revisada por pares e porque essa é a fonte de dados mais comum em análises bibliométricas, como discutido em Mongeon \& Paul-Hus (2016). Com base em uma pesquisa bibliográfica narrativa preliminar sobre o tema, foi identificada e adotada a seguinte combinação de palavras-chave para o processo de busca: (last mile) and (city logistics or urban freight distribution or urban freight transport*) and (operation or best practices or innovation or future). Foi restringida a pesquisa aos anos de 2007 a 2018, por entender que é um tema relativamente novo na literatura, de modo que a maioria das publicações é recente.

Para filtrar os documentos identificados na pesquisa, foram definidos os seguintes critérios de exclusão: artigos que se distanciavam do tema por não serem relativos à etapa de última milha e/ou transporte urbano de carga e não se concentrarem em regiões metropolitanas. Esse critério de exclusão foi aplicado na revisão dos resumos e na revisão de texto completo.

Inicialmente, foram identificados 45 artigos relacionados ao tema. 0 processo de revisão do título e resumo culminou com a seleção de 30 artigos para leitura completa. Seguindo a sistemática prevista na RBS, após a leitura, 23 foram selecionados para serem, de fato, analisados na pesquisa. Os artigos selecionados para leitura completa foram publicados em 12 periódicos científicos diferentes e nos anais de um Congresso Internacional (Tabela 2). 
Tabela 2 - Distribuição dos artigos selecionados por periódico científico

\begin{tabular}{lc}
\hline \multicolumn{1}{c}{ Periódico científico } & Quantidade de artigos \\
\hline Transportation Research Procedia & 4 \\
Transportation Research & 3 \\
Transportation Research Record: Journal of the Transportation Research Board & 2 \\
Journal of Business Logistics & 1 \\
Sustainability & 1 \\
International Journal of Physical Distribution \& Logistics Management & 1 \\
Case Studies on Transportation Policy & 1 \\
Research in Transportation Economics & 1 \\
Procedia - Social and Behavioral Sciences & 1 \\
Transport Policy & 1 \\
International Journal of Sustainable Transportation & 1 \\
Journal of Land Use, Mobility and Environment & 1 \\
International Conference on Ecological Vehicles and Renewable Energies & 1 \\
\hline
\end{tabular}

Fonte: Elaborada pelos autores.

Foi verificado que os artigos analisados foram desenvolvidos em 12 países diferentes e, em sua maioria (82\%), nos últimos três anos, por ser um tema relativamente recente na literatura mundial. A maior concentração de estudos ocorreu na Europa (16), América do Norte (4), América do Sul (1), Oceania (1) e Ásia (1).

As soluções descritas em cada um dos artigos analisados foram armazenadas e organizadas em uma planilha eletrônica, de modo a melhor visualizar as vantagens/desvantagens de cada uma. A seção a seguir abordará os resultados da RBS

Ainda, para reforçar a análise desses resultados, foi desenvolvida uma pesquisa survey com representantes de 293 empresas que atuam no Brasil e realizam o transporte urbano de suas próprias mercadorias ou de terceiros.

0 instrumento de pesquisa adotado na survey foi desenvolvido com base na pesquisa de Joerss et al. (2016) e nos resultados da RBS. Esse instrumento foi estruturado em três blocos: (1) identificação do tipo de produto transportado pelas empresas e seu local de atuação; (2) informações das viagens e principais dificuldades encontradas pelos transportadores; e (3) disposição de cada empresa entrevistada em adotar as práticas inovadoras identificadas na literatura. A validade de face e de conteúdo do questionário foi realizada por três acadêmicos da área de logística e cinco gestores de logística em organizações, que realizavam a distribuição de seus produtos. A confiabilidade do instrumento foi testada utilizando o alfa de Cronbach (alfa $=0,914$ ).

Os questionários foram enviados por e-mail com o link da pesquisa no Google Docs, sendo os dados coletados nos meses de novembro e dezembro de 2018. Esses dados foram consolidados e organizados com o uso de uma planilha eletrônica. A consolidação da base de dados resultou na eliminação de três questionários. Nenhum item apresentou um número expressivo de dados omissos, e não foram detectadas observações atípicas sob a perspectiva univariada (escores $\mathrm{Z}$ das variáveis padronizadas) ou multivariada ( $\mathrm{D}^{2}$ de Mahalanobis). Assim, no total, foram obtidas 38 respostas válidas.

\section{Resultados}

Esta seção, que apresentará os resultados da pesquisa, está dividida em duas subseções: a primeira trata das práticas inovadoras de entrega de última milha identificadas na RBS, enquanto a segunda apresenta a percepção dos respondentes da survey com relação às dificuldades e aos benefícios na introdução das práticas identificadas no contexto de um país em desenvolvimento. 


\section{Resultados da RBS}

Na RBS, foram identificados seis modelos inovadores de entrega de última milha: (1) bicicleta/triciclo elétrico de carga; (2) pontos de coleta; (3) crowdsourcing; (4) drones; (5) entrega na mala do carro $^{1}$; e (6) veículos autônomos. A Tabela 3 mostra a frequência com que cada modelo foi encontrado na pesquisa. Das práticas identificadas, duas não haviam sido abordadas por Joerss et al. (2016): entrega na mala do carro e pontos de coleta. Além disso, pela sua semelhança e analogia, foi feita a opção por juntar as práticas droide e veículos semiautônomos, apontadas por Joerss et al. (2016), em uma única categoria: veículos autônomos ou semiautônomos.

Tabela 3 - Quantidade de vezes em que cada modelo foi encontrado nos artigos selecionados

\begin{tabular}{clc}
\hline $\mathbf{N}^{\mathbf{0}}$ & \multicolumn{1}{c}{ Modelo de entrega } & Quantidade de artigos \\
\hline 1 & Bicicleta/triciclo elétrico de carga & 5 \\
2 & Crowdsourcing & 2 \\
3 & Drones & 2 \\
4 & Entrega na mala do carro & 1 \\
5 & Pontos de coletal & 8 \\
6 & Veículos semiautônomos ou autônomos ${ }^{2}$ & 1 \\
\hline
\end{tabular}

IIncluem armários de encomenda, entrega no local de trabalho. IIncluem veículos autônomos, semiautônomos e droides.

Por meio da análise dos artigos, foi identificada, com a adoção de cada modelo de entrega, a redução: do consumo de combustível fóssil, da emissão de PA e de GEE, da geração de ruído, dos custos na entrega de última milha, da distância percorrida pelo transportador, de congestionamento, de viagens perdidas e do tempo de transporte. Ainda, foram citados benefícios como aumento da segurança viária, maior flexibilidade de contratação de motorista, maior flexibilidade de coleta e devolução por parte do cliente e maior flexibilidade de entrega por parte do transportador. A Tabela 4 apresenta os benefícios identificados com maior frequência para cada modelo inovador de entrega.

Tabela 4 - Benefícios identificados em cada modelo de entrega

\begin{tabular}{|c|c|c|c|c|c|c|c|c|}
\hline Modelo inovador de entrega & 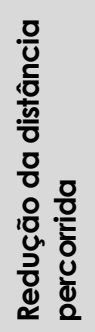 & 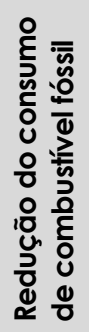 & 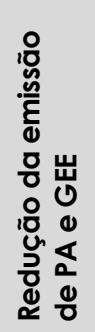 & 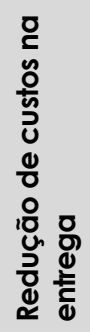 & 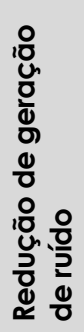 & 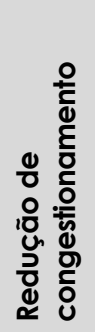 & 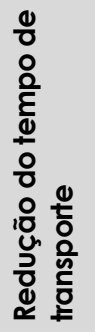 & 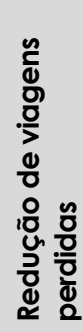 \\
\hline Bicicleta/triciclo elétrico de carga & $x$ & $x$ & $\mathrm{x}$ & $x$ & $\mathrm{x}$ & $x$ & $\mathrm{x}$ & \\
\hline Crowdsourcing & & & & $x$ & & & & \\
\hline Drones & $x$ & & & & $x$ & $x$ & $x$ & \\
\hline Entrega na mala do carro & $x$ & $x$ & $x$ & & & & & $x$ \\
\hline Pontos de coleta & $x$ & $x$ & $x$ & $x$ & $x$ & $x$ & $x$ & $x$ \\
\hline Veículos autônomos & & $x$ & $x$ & & $x$ & & & \\
\hline
\end{tabular}

Do total de artigos, 65\% sinalizaram a redução de PA e GEE ou do consumo de combustíveis fósseis como os principais benefícios ambientais, além da redução da geração de ruído. Ainda, 48\% propuseram iniciativas baseadas em propulsão humana como principal fonte de energia, 30\% incentivaram a eletromobilidade e $22 \%$ ainda indicaram alternativas dependentes de combustíveis fósseis, como gasolina e diesel.

\footnotetext{
${ }^{1}$ As entregas são realizadas diretamente na mala dos veículos dos consumidores, sendo utilizada uma chave digital de uso único por meio da qual os transportadores conseguem desbloquear o veículo do cliente (Reyes et al., 2017). Esse serviço está disponível nos Estados Unidos com o nome de CarDrops.
} 
Os principais óbices relacionados à adoção dos novos modelos de entrega identificados na literatura foram: limitação de dimensões e peso de mercadorias a serem entregues, limitação de distância percorrida, restrição de velocidade, necessidade de infraestrutura urbana, área densamente povoada e limitação de segurança. No caso dos modelos que adotam veículos elétricos, também foram apontados como obstáculos: alto custo de aquisição, alto custo da bateria, baixa autonomia da bateria, necessidade de infraestrutura de recarga, carência de recursos humanos especializados e longo tempo de recarga da bateria. A Tabela 5 apresenta os principais óbices de cada modelo de entrega.

Tabela 5 - Óbices de cada modelo de entrega

\begin{tabular}{|c|c|c|c|c|c|c|}
\hline Prática & 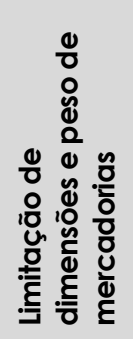 & 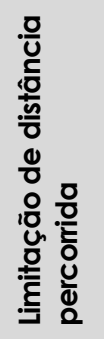 & 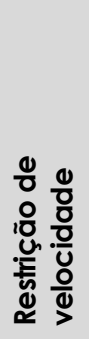 & 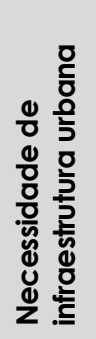 & 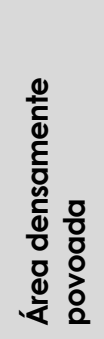 & 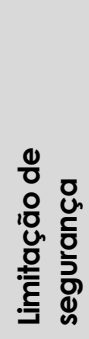 \\
\hline Bicicleta/triciclo elétrico de carga & $\bar{x}$ & $\bar{x}$ & $\bar{x}$ & $\bar{x}$ & $\bar{x}$ & \\
\hline Crowdsourcing & $\mathrm{x}$ & & & & & \\
\hline Drones & $\mathrm{x}$ & $\mathrm{x}$ & $\mathrm{x}$ & & & $\mathrm{x}$ \\
\hline Entrega na mala do carro & $\mathrm{x}$ & & & & & $\mathrm{x}$ \\
\hline Pontos de coleta & $x$ & & & & $x$ & $\mathrm{x}$ \\
\hline Veículos autônomos & $x$ & & & $\mathrm{x}$ & $\mathrm{x}$ & $\mathrm{x}$ \\
\hline
\end{tabular}

É observado que $83 \%$ dos trabalhos revisados abordaram práticas com alguma restrição de tamanho de carga útil, o que reflete uma tendência à adoção de veículos de tamanho reduzido e iniciativas com baixa capacidade de armazenagem, próprios para áreas densamente povoadas, tal como reforçado por Gevaers et al. (2014). Assim, a restrição no tamanho de encomendas seria o principal óbice dos novos modelos de entrega, apontando que a maioria das soluções inovadoras para a última milha seria indicada para pequenas mercadorias e poucas entregas por viagem. Embora seja uma desvantagem das práticas abordadas, é percebido que esse perfil de pequenas mercadorias e de poucas entregas por viagem é o que melhor representa a realidade brasileira no que se refere à última milha, como será discutido na seção 5 .

Survey

A Figura 1 apresenta o mercado de atuação das empresas entrevistadas. É destacado que $44,4 \%$ das empresas analisadas eram organizações industriais, 38,9\% atuavam na área de transportes, e 22,2\%, no segmento de varejo. Ainda, as empresas consultadas atuavam em áreas bastante diversificadas. Por meio da Figura 1, é verificado que, em sua maioria, essas empresas comercializavam produtos de pequeno porte, como eletrodomésticos, alimentos e comércio eletrônico. Ademais, 94\% das empresas consultadas se situavam, principalmente, na região Sudeste do país, onde estão concentrados os principais polos industriais brasileiros, bem como a maioria da população nacional (IBGE, 2010). 


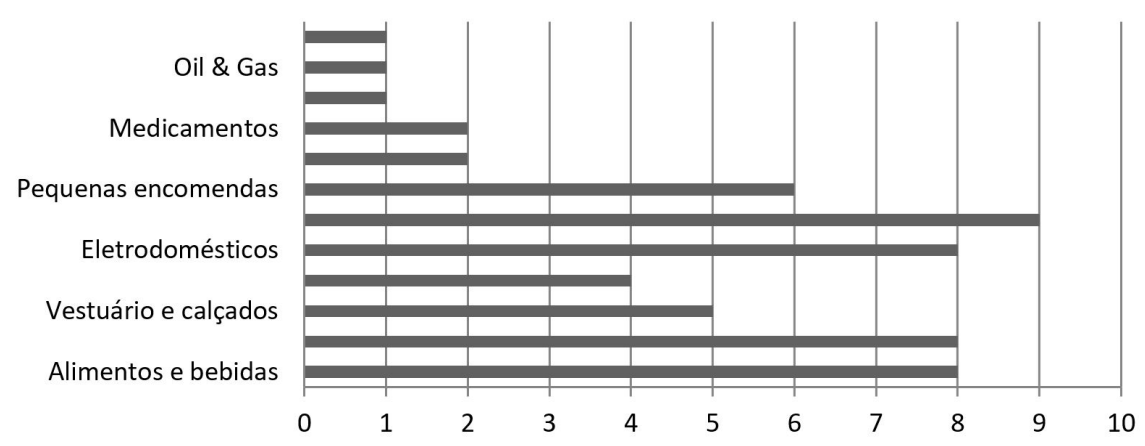

Figura 1 - Classe dos produtos transportados pelas empresas.

Em média, as empresas entrevistadas realizavam 16 entregas por viagem, sendo verificado um mínimo de 1 e máximo de 40 entregas por viagem. Isso confirma o fato de serem produtos de pequeno porte os mais frequentes nas entregas de última milha. Quanto aos locais de entrega das mercadorias, $72 \%$ eram realizados em estabelecimentos comerciais, presentes nas áreas mais urbanizadas das cidades, enquanto $11 \%$ eram residenciais, e 17\%, em ambos. Além disso, $67 \%$ das entregas de carga na última milha eram realizadas em áreas centrais das cidades.

Quanto às principais dificuldades presentes na entrega das mercadorias, as empresas relataram que as seguintes barreiras ocorriam com muita frequência: falta de segurança (44\%), congestionamentos (33\%), falta de vaga para estacionamento (28\%), uso inadequado de vagas de carga/descarga (11\%) e viagem perdida pelo não recebimento da carga (6\%). Além dessas, outras dificuldades também foram citadas pelos transportadores, como áreas restritas à passagem de caminhões, tempo de carga/descarga elevado, entregas em áreas de risco, restrições ao tamanho do veículo utilizado para entrega e limitação de acesso por rodízio de placas.

As empresas entrevistadas também responderam acerca da disposição de cada uma em adotar as práticas inovadoras identificadas na literatura, bem como os principais óbices e benefícios considerados nessa adoção. As subseções a seguir apresentarão os resultados obtidos para cada prática identificada na RBS.

\section{Bicicleta/triciclo elétrico}

A Figura 2 mostra a distribuição das respostas quanto à possibilidade de realização da distribuição de mercadorias em áreas urbanas por meio de bicicletas/triciclos elétricos. Como a maioria dos produtos era de pequeno porte, os transportadores consideraram viável seu transporte via bicicleta, visto que $44 \%$ das respostas foram favoráveis ao seu uso e $17 \%$ eram possivelmente favoráveis. Ao considerar apenas as respostas nas quais o produto transportado permitia o uso de bicicletas, cerca de $80 \%$ das respostas foram favoráveis ou possivelmente favoráveis a seu uso.

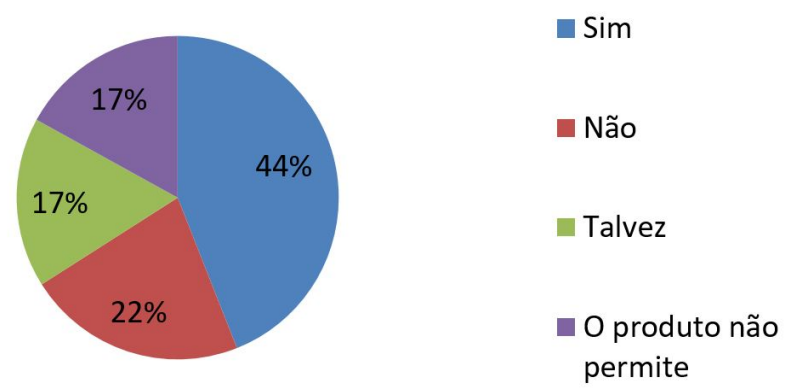

Figura 2 - Distribuição das respostas das empresas quanto ao uso de bicicleta/triciclo elétrico. 
Além disso, os entrevistados, em sua maioria (61\%), concordaram que o uso de bicicletas/triciclos elétricos seria uma iniciativa que reduziria a emissão de PA e de GEE. Ainda, 28\% acreditavam que essa iniciativa diminuiria os custos de entrega. Em relação aos óbices relacionados à adoção das práticas, $67 \%$ dos entrevistados afirmaram que, no caso de veículos elétricos, o maior desafio seria a falta de infraestrutura para recarga das baterias e a falta de legislação que regulamentasse seu uso. De uma forma geral, consideraram a falta de ciclovias ou vias preferenciais como barreiras à introdução dessa prática atualmente no Brasil.

\section{Pontos de coleta}

A Figura 3 mostra a distribuição das respostas dadas pelas empresas entrevistadas a respeito do uso de pontos de coleta para posterior retirada da mercadoria pelo consumidor. É possível perceber que nenhuma empresa descartou o uso dessa prática, apenas consideraram que seu produto não permitia essa adoção.

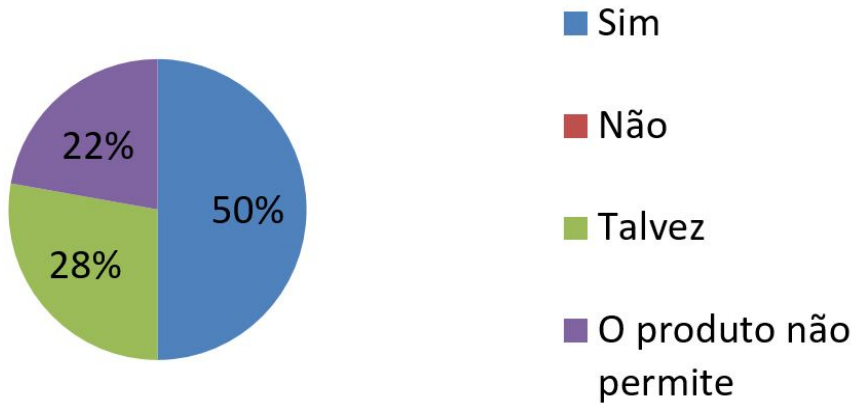

Figura 3 - Distribuição das respostas das empresas entrevistadas quanto ao uso de pontos de coleta.

Em relação aos benefícios, $61 \%$ dos entrevistados acreditavam que a redução do tempo de entrega seria alcançada com a adoção dessa prática. Por outro lado, 56\% e $61 \%$ achavam que a diminuição de viagens perdidas e a maior flexibilidade para o consumidor recolher sua mercadoria, respectivamente, seriam vantagens com grande probabilidade de ocorrência. Economia de combustível também foi citado por $56 \%$ dos entrevistados como vantagem que muito provavelmente seria alcançada. Por sua vez, 50\% dos entrevistados acreditavam na redução da emissão de PA e GEE como vantagem com maior probabilidade de ocorrer.

Os entrevistados não apresentaram um consenso com relação aos desafios que a adoção dessa prática poderia enfrentar. Dos respondentes, $28 \%$ acreditavam que o aumento do custo do transporte seria uma desvantagem com baixa probabilidade de acontecer, $17 \%$, um desafio com mais alta probabilidade de ocorrência, e 5\% não achavam que isso aconteceria. Em relação à falta de segurança, $55 \%$ consideravam que seria recorrente ou muito recorrente problemas desse tipo em sua área.

Quando questionados sobre a satisfação do cliente com o uso dessa iniciativa, 67\% das empresas achavam que o cliente ficaria pouco satisfeito ou apenas satisfeito com o novo modelo de entrega. Já em relação à disponibilidade de estabelecimentos comerciais para abrigar os pontos de coleta, $28 \%$ acreditavam que muito provavelmente isso seria um grande desafio ao adotar essa iniciativa.

A partir das respostas descritas, é constatado que, apesar de a adoção de pontos de coleta ser uma prática com certa "simpatia" pelas empresas, estas estavam cientes dos desafios que essa iniciativa poderia gerar e, mesmo assim, achavam que seu uso seria viável no cenário brasileiro. 0 fato de a maioria ter relatado a redução do tempo de entrega como principal benefício reforça a preocupação com os congestionamentos e a falta de vaga de estacionamento. 


\section{Crowdsourcing}

A Figura 4 mostra a distribuição das respostas dadas pelas empresas em relação à contratação de um entregador independente para realizar entregas de última milha por meio de crowdsourcing. É possível perceber que as respostas ficaram quase igualmente distribuídas, o que pode indicar indecisão dos entrevistados ou não conhecimento suficiente sobre a prática crowdsourcing.

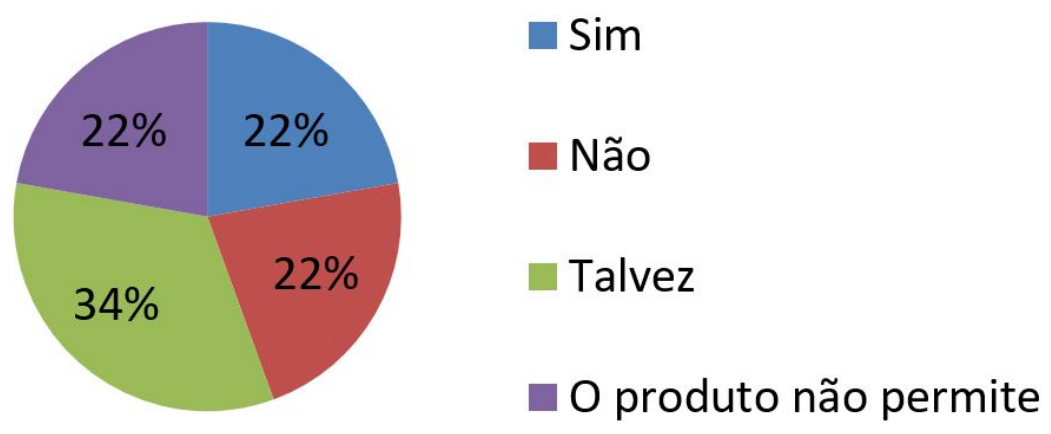

Figura 4 - Distribuição das respostas das empresas entrevistadas quanto ao uso de crowdsourcing.

Em relação às vantagens que essa prática poderia gerar, 55\% acreditavam que a geração de novos empregos para motoristas seria uma vantagem observada, enquanto $44 \%$ achavam que o menor gasto com manutenção e aquisição de veículos seria plenamente observado. Já quanto aos desafios dessa iniciativa, 56\% consideravam que o principal obstáculo que impedia a introdução dessa modalidade seria a falta de regulamentação para esse tipo de transporte, especialmente no que se concerne a questões fiscais e contratuais, como responsabilidades referentes a danos na carga etc.

\section{Veículos semiautônomos e autônomos}

A Figura 5 mostra a distribuição das respostas a respeito da adoção de veículos autônomos para o transporte de carga na última milha. Assim como no caso da utilização de crowdsourcing, as respostas foram bem distribuídas, indicando, assim, um possível desconhecimento a respeito do uso de veículos autônomos por parte dos entrevistados.

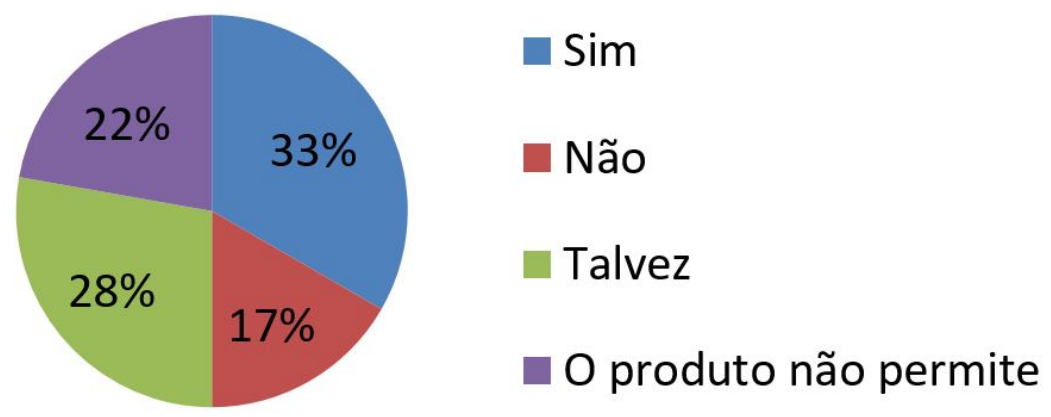

Figura 5 - Distribuição das respostas quanto ao uso de veículos autônomos.

Quanto aos benefícios que tal prática poderia gerar, $50 \%$ dos entrevistados apontaram o fato de não precisar limitar a jornada de trabalho a 8 horas por dia para um veículo autônomo, diferentemente de um trabalhador comum, o que geraria maior produtividade. Além disso, 33\% acreditavam que a redução no número de funcionários e o fim da necessidade de treinar motoristas seriam vantagens que 
muito provavelmente aconteceriam. Porém, 39\% dos entrevistados afirmaram que entregas mais rápidas seriam uma vantagem pouco provável ou com nenhuma probabilidade de acontecer.

Em relação aos obstáculos, $61 \%$ dos entrevistados indicaram o alto custo de aquisição do veículo autônomo como principal barreira para seu uso. A falta de regulamentação própria e a falta de investimentos nessa tecnologia foram citadas por $56 \%$ dos entrevistados como desafios que muito provavelmente impediriam o seu uso no Brasil.

A partir das respostas dos entrevistados, é percebido o potencial da adoção de veículos autônomos para última milha em países em desenvolvimento. No entanto, fica condicionada à necessidade de maior investimento nessa tecnologia. Uma grande parcela dos entrevistados não rejeitou seu uso, e houve uma convergência entre os desafios dessa prática, o que indica que essas empresas são potenciais usuárias dessa nova prática.

\section{Drones}

A Figura 6 traz a distribuição das respostas a respeito da utilização de drones para a última milha. Dos entrevistados, $33 \%$ rejeitaram o uso desse tipo de transporte, enquanto $22 \%$ afirmaram que o tipo de produto de sua empresa não permitia seu uso. Ao analisar a Figura 6, é constatado que 67\% das empresas que poderiam utilizar drones para realizar o transporte de última milha de suas mercadorias consideravam viável ou talvez viável sua adoção. Os entrevistados indicaram a viabilidade do uso de drones para entregas de última milha por causa da pequena dimensão de suas mercadorias.

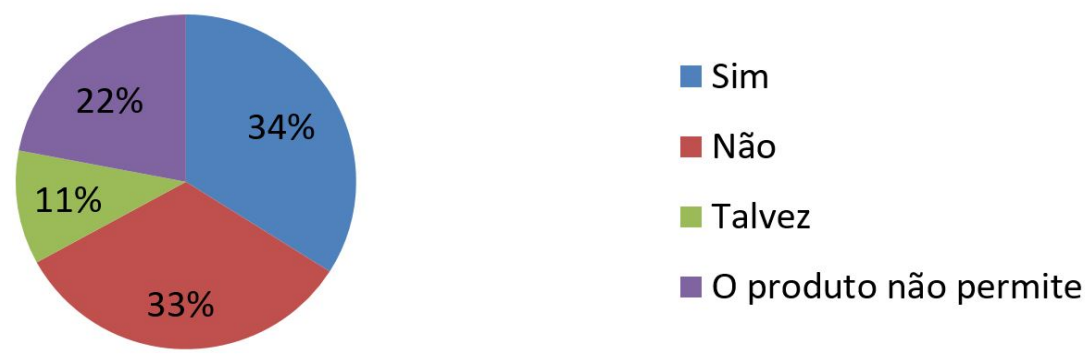

Figura 6 - Distribuição das respostas das empresas entrevistadas quanto ao uso de drones.

Quanto aos benefícios de tal prática, 50\% dos entrevistados apontaram maior rapidez na entrega, redução no consumo de combustível e redução da emissão de PA e GEE como os principais benefícios dessa iniciativa. No que se refere aos obstáculos possíveis, a falta de legislação apropriada (61\%) e o alto custo dos equipamentos (50\%) foram considerados os mais possíveis de ocorrer. A possibilidade de acidentes (33\%) e o volume reduzido de carga transportada (39\%) também foram citados como obstáculos recorrentes ao adotar drones na última milha do transporte de carga.

\section{Entrega na mala do carro}

A Figura 7 mostra a distribuição das respostas a respeito da realização de entregas na mala do carro do cliente, com o auxílio de rastreador para acompanhar a localização em tempo real do veículo. É constatado que 33\% das empresas entrevistadas rejeitavam a utilização dessa prática, mas 56\% delas se mostravam adeptas ou ao menos consideravam a possibilidade do seu uso, dependendo da circunstância. 


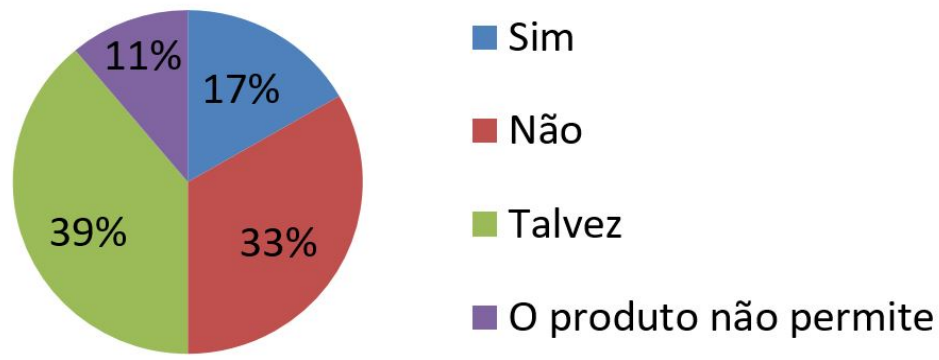

Figura 7 - Distribuição das respostas quanto a entregas na mala do carro.

Cerca de $40 \%$ dos entrevistados afirmaram que a redução no número de entregas não realizadas e a maior flexibilidade para o transportador realizar as entregas seriam os principais benefícios que essa prática poderia gerar. Porém, $40 \%$ dos respondentes apontaram que a falta de confiança por parte do cliente nessa modalidade de entrega seria a principal barreira para sua adoção. Ainda, 33\% acreditavam que os altos índices de criminalidade e a tecnologia insuficiente no Brasil seriam obstáculos que muito provavelmente impediriam seu uso no cenário brasileiro.

Enfim, a Tabela 6 sintetiza os resultados da pesquisa survey, apresentando a distribuição das respostas a cada prática inovadora analisada. É possível perceber que a adoção de pontos de coleta, além de representar a modalidade com maior aceitação pelos respondentes $(50 \%$ afirmaram que utilizariam tal modalidade), também foi a única que não teve nenhuma rejeição, com isso apresentando um alto potencial de sucesso. Por outro lado, a entrega na mala do carro e os drones foram os que apresentaram maior rejeição (33\%). A seção a seguir apresentará a análise dos resultados obtidos.

Tabela 6 - Resumo das respostas obtidas

\begin{tabular}{|c|c|c|c|c|}
\hline Práticas / respostas & Sim & Não & Talvez & $\begin{array}{c}\text { O produto não } \\
\text { permite }\end{array}$ \\
\hline Bicicleta/triciclo elétrico & $44 \%$ & $22 \%$ & $17 \%$ & $17 \%$ \\
\hline Pontos de coleta & $50 \%$ & $0 \%$ & $28 \%$ & $22 \%$ \\
\hline Crowdsourcing & $22 \%$ & $22 \%$ & $34 \%$ & $22 \%$ \\
\hline Veículos semiautônomos e autônomos & $33 \%$ & $17 \%$ & $28 \%$ & $22 \%$ \\
\hline Drones & $34 \%$ & $33 \%$ & $11 \%$ & $22 \%$ \\
\hline Entrega na mala do carro & $17 \%$ & $33 \%$ & $39 \%$ & $11 \%$ \\
\hline
\end{tabular}

\section{Análise dos resultados}

Nesta seção, será realizada uma discussão sobre os resultados da pesquisa, em especial comparando aqueles obtidos pela survey com os da RBS. Além disso, a intenção foi avaliar como o nível de desenvolvimento econômico de um país onde a prática é aplicada influencia sua adoção e, principalmente, suas vantagens e desvantagens. Assim sendo, a Tabela 7 apresenta uma consolidação das práticas identificadas na RBS e analisadas na pesquisa survey, destacando aquelas que foram consideradas, por parte das empresas analisadas, viáveis para aplicação no cenário das cidades brasileiras.

Tabela 7 - Resumo dos resultados obtidos na pesquisa de mercado

\begin{tabular}{cc}
\hline Práticas inovadoras & $\begin{array}{c}\text { Viável para o cenário brasileiro de acordo com a } \\
\text { pesquisa de mercado? }\end{array}$ \\
\hline Bicicleta/triciclo elétrico & $\mathrm{Sim}$ \\
Pontos de coleta & $\mathrm{Sim}$ \\
Crowdsourcing & $\mathrm{Sim}$ \\
Veículos semiautônomos e autônomos & $\mathrm{Sim}$ \\
Drones & $\mathrm{Sim}$ \\
Entrega na mala do carro & $\mathrm{Não}$ \\
\hline
\end{tabular}

Fonte: Elaborada pelos autores. 
0 resultado da survey em relação à adoção da prática bicicletas/triciclos elétricos mostra as empresas, em sua maioria, que realizavam transporte na última milha eram conscientes acerca dos benefícios e desafios que essa iniciativa poderia gerar, facilitando, assim, sua adoção. Ainda, reforçaram que essa prática era adequada ao cenário brasileiro, apesar de ainda apresentar desafios que precisam ser superados. Para os entrevistados, o maior desafio que impediria essa prática de ser amplamente adotada na realidade brasileira era a questão da infraestrutura urbana, tal como indicado por Morganti \& Browne (2018). Porém, para o cenário de países desenvolvidos, Morganti et al. (2015) identificaram como desafios a recente tecnologia das baterias e a falta de variedade de veículos no mercado ao considerar o uso de bicicletas ou triciclos elétricos.

Quanto à prática ponto de coleta, a redução do tempo de entrega foi um benefício identificado na survey. A redução do número de viagens perdidas e a maior flexibilidade para o consumidor recolher sua mercadoria também foram identificadas como vantagens, tal como abordado por Oliveira et al. (2017). Outras vantagens identificadas na survey foram economia de combustível e redução da emissão de PA e GEE. Além disso, Brummelman et al. (2003) observaram que pontos de coleta, quando instalados em locais de grande movimentação de pessoas, geravam a oportunidade de o cliente recolher suas mercadorias durante seu percurso diário, sem gerar uma nova viagem. Foi constatada uma divergência entre os resultados da literatura e da survey em relação ao custo da adoção dessa prática, sendo visto como alto na survey.

No que se refere à prática crowdsourcing, as vantagens e desvantagens percebidas pelas empresas entrevistadas convergiram com o que foi encontrado na literatura. Foi identificado que esse modelo distribuía custos e benefícios entre os envolvidos, porém carecia de regulamentação. Ainda, Allen et al. (2017) destacaram ser possível transportar mercadorias em uma mesma viagem com uma significativa vantagem. Entretanto, a falta de propagação dessa modalidade no cenário brasileiro foi evidenciada ao observar a predominância de artigos europeus sobre o tema na literatura. Com isso, é possível dizer que o crowdsourcing é uma prática com potencial para ser introduzida no cenário brasileiro, mas que ainda necessita de maior divulgação entre os gestores no Brasil, além de requerer investimentos iniciais e definições com relação a questões fiscais e contratuais.

No que concerne aos veículos autônomos, as vantagens e desvantagens citadas pelos entrevistados convergiram com as aquelas identificadas na literatura, principalmente com Pal \& Kant (2016). Esses autores consideraram que o alto custo desse tipo de transporte seria o principal limitador para o uso, enquanto o fato de não ser necessário treinar motoristas seria a principal vantagem observada. Ainda, 0 resultado da survey indicou um possível desconhecimento a respeito do uso desse tipo de veículo, já que essa tecnologia ainda está iniciando no cotidiano brasileiro. Entre as desvantagens, os elevados índices de criminalidade e a possibilidade de acidentes não foram considerados como obstáculos significantes.

Em relação aos drones, a maioria das empresas entrevistadas consideraram ser viável a sua adoção na última milha, por ser uma alternativa adequada para o transporte de pequenas mercadorias. Ranieri et al. (2016) e Heutger \& Kuckelhaus (2017) reforçaram que a rapidez na entrega seria a principal vantagem observada nessa prática, porém apontaram o congestionamento aéreo e a limitação do tamanho da carga transportadas como as principais barreiras para essa prática. Por sua vez, as empresas entrevistadas indicaram a falta de legislação apropriada e o alto custo dos equipamentos como os principais desafios. Essa diferença se deve ao fato de que os autores Ranieri et al. (2016) e Heutger \& Kuckelhaus (2017) são de países desenvolvidos (Itália e Estados Unidos, respectivamente), onde esse tipo de tecnologia já é mais desenvolvido, e, por isso, drones não são considerados equipamentos de alto custo. Ainda assim, uma boa parcela das empresas que realizavam a última milha no Brasil demonstrou interesse na utilização de drones.

Quanto à entrega na mala do carro, apenas Reyes et al. (2017) abordaram essa prática inovadora para a última milha, o que reforça que essa modalidade é pouco explorada até mesmo na literatura. Tanto as empresas entrevistadas quanto Reyes et al. (2017) consideraram que a principal vantagem era a maior flexibilidade para o transportador realizar entregas. Porém, as respostas da survey indicaram que tal prática inovadora não parecia viável à realidade brasileira, visto que grande parte dos 
transportadores não se mostrou confiante com a alternativa e convergiu para obstáculos comuns, como falta de confiança por parte do cliente e altos índices de criminalidade. Considerando que, até o momento, esse tipo de entrega é realizado apenas em países desenvolvidos, os desafios para adoção dessa prática estão relacionados aos problemas enfrentados por países em desenvolvimento como o Brasil.

\section{Conclusão}

Este trabalho foi capaz de identificar novas formas de operação adotadas na última milha por meio de uma RBS de abrangência internacional, acompanhada de uma survey que procurou complementar os resultados e direcioná-los para a realidade de um país em desenvolvimento, no caso o Brasil. Entre essas formas de operação destacam-se: o emprego de bicicletas e triciclos elétricos de carga, o uso de drones, o emprego de veículos autônomos, o crowdsourcing, a entrega diretamente na mala do carro e a entrega em pontos de coleta.

A combinação da aplicação da RBS com a survey possibilitou compreender melhor os requisitos necessários para a introdução dessas formas de operação, em particular em contexto de um país em desenvolvimento, destacando os benefícios e os desafios relacionados a esse processo.

É percebido, por meio dos resultados da RBS, que países mais desenvolvidos economicamente tendem a buscar soluções mais sofisticadas para a última milha, com uso de veículos tecnologicamente inovadores, visando, principalmente, reduzir o emprego de mão de obra e os impactos ambientais e melhorar a eficiência dessa etapa do transporte. Por sua vez, os países menos desenvolvidos economicamente apostam em soluções com menor nível de tecnologia associada, de fácil adoção e com baixa necessidade de investimentos financeiros, pois ainda precisam enfrentar outros desafios recorrentes. Ademais, a redução do emprego de mão de obra não foi verificada como um objetivo a ser alçando nos países em desenvolvimento por meio da aplicação de novas formas de operação de entregas, visto que o custo de mão de obra nesses países ainda é inferior em comparação ao de países desenvolvidos. Esse resultado foi corroborado no contexto brasileiro pelos resultados da pesquisa survey.

Foi identificado na revisão bibliográfica que a Europa é o continente que mais realiza pesquisas sobre o tema em questão, seguido da América do Norte. Tal fato representou uma limitação durante a análise das vantagens e desvantagens de cada prática, considerando o nível de desenvolvimento econômico de cada país, já que a maioria (90\%) dos artigos utilizados é proveniente de países desenvolvidos. A pesquisa de mercado com empresas que realizam o transporte de carga de última milha no Brasil possibilitou contornar essa dificuldade. A partir das respostas baseadas na disposição de cada empresa em adotar as práticas, foi possível identificar quais delas têm potencial para ser introduzidas no cenário brasileiro. Além disso, como recomendação para trabalhos futuros, é sugerida a realização da análise do ciclo de vida das práticas encontradas, bem como a identificação e a análise de práticas inovadoras em outras etapas do transporte de carga que não sejam as de última milha.

\section{Referências}

Allen, J., Piecyk, M., \& Piotrowska, M. (2017). An analysis of online shopping and home delivery in UK: Projeto Freight Traffic Control 2050. London: Universidade de Westminster.

Brummelman, H. J., Kuipers, B., \& Vale, N. (2003). Impacts of locker points on mobility. Delft: TNO Inro.

Comi, A., \& Nuzzolo, A. (2016). Exploring the relationships between e-shopping attitudes and urban freight transport. Transportation Research Procedia, 12, 399-412. http://dx.doi.org/10.1016/j.trpro.2016.02.075. 
Gevaers, R., Van De Voorde, E., \& Vanelslander, T. (2014). Cost modelling and simulation of last-mile characteristics in an innovative B2C supply chain environment with implications on urban areas and cities. Procedia: Social and Behavioral Sciences, 125, 398-411. http://dx.doi.org/10.1016/j.sbspro.2014.01.1483.

Heutger, M., \& Kuckelhaus, M. (2017). Unmanned aerial vehicles in logistics: a DHL perspective on implications and use for the logistics industry. Troisdorf: DHL Customer Solutions \& Innovation. Recuperado em 2 de novembro de 2018, de https://workhorse.com /newsroom/2017/02/ups-tests-residential-delivery-drone

Instituto Brasileiro de Geografia e Estatística - IBGE. (2010). Séries históricas e estatísticas. Rio de Janeiro: IBGE. Recuperado em 28 de dezembro de 2018, de https://seriesestatisticas.ibge.gov.br/series.aspx?no=10\& op $=0 \&$ vcodigo $=$ POP100\& $\mathrm{t}=$ populacao-residente-distribuicao-grandes-regioes

Joerss, M., Schröder, J., Neuhaus, F., Klink, C., \& Mann, F. (2016). Parcel delivery: the future of last mile. New York: McKinsey \& Company. Recuperado em 27 de julho de 2017, de http://www.mckinsey.com/industries/traveltransport-and-logistics/our-insights/how-customerdemands-are-reshaping-last-mile-delivery

Lindholm, M., \& Behrends, S. (2012). Challenges in urban freight transport planning: a review in the Baltic Sea Region. Journal of Transport Geography, 22, 129-136. http://dx.doi.org/10.1016/j.jtrangeo.2012.01.001.

McKinnon, A. C., Browne, M., \& Whiteing, A. E. (2015). Green logistics: improving the environmental sustainability of logistics. London: Kogan Page Publishers.

Mongeon, P., \& Paul-Hus, A. (2016). The journal coverage of Web of Science and Scopus: a comparative analysis. Scientometrics, 106(1), 213-228. http://dx.doi.org/10.1007/s11192-015-1765-5.

Morganti, E., Boutueil, V., \& Leurent, F. (2015). BEVs and PHEVs in France: market trends and key drivers of their short-term development (Working Papers). Paris: Paristech. Dipsonível em: https://hal-enpc.archivesouvertes.fr/hal-01294644/document. Acesso em Julho de 2019.

Morganti, E., \& Browne, M. (2018). Technical and operational obstacles to the adoption of electric vans in France and the UK: an operator perspective. Transport Policy, 63, 90-97. http://dx.doi.org/10.1016/j.tranpol.2017.12.010.

Muñoz-Villamizar, A., Montoya-Torres, J. R., \& Faulin, J. (2017). Impact of the use of electric vehicles in collaborative urban transport networks: a case study. Transportation Research Part D, Transport and Environment, 50, 40-54. http://dx.doi.org/10.1016/j.trd.2016.10.018.

Oliveira, L. K., Morganti, E., Dablanc, L., \& Oliveira, R. L. M. (2017). Analysis of the potential demand of automated delivery stations for e-commerce deliveries in Belo Horizonte, Brazil. Research in Transportation Economics, 65, 34-43. http://dx.doi.org/10.1016/j.retrec.2017.09.003.

Pal, A., \& Kant, K. (2016). A combined perishable food and people transport architecture in smart urban areas. In IEEE International Conference on Smart Computing. USA: IEEE.

http://dx.doi.org/10.1109/SMARTCOMP.2016.7501716.

Ranieri, L., Digiesi, S., \& Silvestri, B. (2016). A review of last mile logistics innovations in an externalities cost reduction vision. Sustainability, 10(3), 1-18.

Reyes, D., Savelsbergh, M., \& Toriello, A. (2017). Vehicle routing with roaming delivery locations. Transportation Research Part C, Emerging Technologies, 80, 71-91. http://dx.doi.org/10.1016/j.trc.2017.04.003.

Roumboutsos, A., Kapros, S., \& Vanelslander, T. (2014). Green city logistics: systems of innovation to assess the potential of E-vehicles. Research in Transportation Business \& Management, 11, 43-52. http://dx.doi.org/10.1016/j.rtbm.2014.06.005.

Sanchez Rodrigues, V., Piecyk, M., Mason, R., \& Boenders, T. (2015). The longer and heavier vehicle debate: a review of empirical evidence from Germany. Transportation Research Part D, Transport and Environment, 40, 114131. http://dx.doi.org/10.1016/j.trd.2015.08.003.

Thomé, A. M., Scavarda, L. F., \& Scavarda, A. J. (2016). Conducting systematic literature review in operations management. Production Planning and Control, 27(5), 408-420.

http://dx.doi.org/10.1080/09537287.2015.1129464. 
United Nations. (2014). World urbanization prospects: the 2014 revision. United Nations Report. New York. Recuperado em 28 de agosto de 2018, de https://esa.un.org/unpd/wup/publications/files/wup2014-

highlights.pdf

Winkenbach, M., Roset, A., \& Spinler, S. (2016). Strategic redesign of urban mail and parcel networks at la poste strategic redesign of urban mail and parcel networks at la poste. Interfaces, 46(5), 445-458. http://dx.doi.org/10.1287/inte.2016.0854.

Editor: Fábio Duarte

Recebido: Jun. 01, 2019

Aprovado: Dez. 15, 2019 